\title{
IV. ALPHA-PARTICLE PHYSICS IN BPX
}

\author{
D. J. SIGMAR (MIT)
}

\section{IV.A. INTRODUCTION}

A central part of the mission of the Burning Plasma Experiment (BPX) is to determine the alpha particle dynamics of self-heated fusion plasmas. While an increasing experimental and theoretical effort exists to simulate alpha-particle physics conditions, BPX will be the first fusion plasma in which the alpha power, $P_{\alpha}$, exceeds the auxiliary heating power, $P_{a u x}$. A second critical condition realized in BPX will come from the existence of relatively large amounts $\left(\beta_{\alpha} \gtrsim 0.2 \%\right)$ of alpha particles with $v_{\|} \geq v_{\text {Alfuen }}$ capable of Cherenkov excitation of the Alfvén spectrum of collective oscillations, which can affect fast alpha confinement. The exploration of this virgin territory can be expected to reveal unanticipated plasma physics phenomena favorably and unfavorably affecting overall performance, just as was the case when the tokamak program moved from ohmically heated to neutral beam and radio-frequency (RF) heated plasmas. Thus, the range of alphaparticle physics results from BPX promises to be of fundamental importance for any subsequent Experimental Test Reactor (ETR).

The list of currently conceptualized alphaparticle physics issues starts with the global questions of (a) what is the self-heating efficiency $\eta_{\alpha}$ with which the alpha power at birth couples to the bulk plasma; (b) particularly in the regime $P_{\alpha}>P_{\text {aux }}$, how does $P_{\alpha}$ affect bulk plasma stability and confinement; (c) what is the central confinement time of the thermalized alpha particles (helium ash); and (d) what are the thermal stability boundaries in plasma operation space including the synergistic alpha effects contained in (a),(b), and (c) above.

The remainder of this section will develop some of these global issues in more detail and analyze the implications for BPX.

\section{IV.B. ENERGETIC ALPHA.PARTICLE COUPLING EFFICIENCY $\eta_{\alpha}$}

Before going into the details of specific loss mechanisms, it is valuable to assess the impact of reduced coupling of the alpha power $\left(P_{\alpha o} \equiv S_{f} E_{\alpha o}\right.$, $\left.S_{f} \simeq\left\langle\sigma_{f} v\right\rangle n_{e}^{2} / 4\right)$ to the background plasma. Given an anomalous $D_{\alpha}$ and ensuing fast alpha loss frequency $\nu_{\mathrm{L}} \sim D_{\alpha} / a^{2}$, it follows readily from al- pha power balance that the coupling parameter $\eta_{\alpha} \equiv\left[P_{\alpha}\right.$ (coupled) $\left./ P_{\alpha o}\right]$ is given by

$$
\begin{aligned}
& \eta_{\alpha}=1-\nu_{L} n_{\alpha} \bar{E}_{\alpha} /\left(S_{f} E_{\alpha o}\right) \\
& n_{\alpha} \bar{E}_{\alpha}=\int_{0}^{v_{o \alpha}} d^{3} v \frac{m_{\alpha} v^{2}}{2} f_{\alpha}
\end{aligned}
$$

and we note that in steady state $S_{f} / n_{\alpha}=1 / \tau_{S D}$. $n_{\alpha} \bar{E}_{\alpha}$ is the velocity distribution $\left(f_{\alpha}\right)$ averaged alpha pressure including the effect of $\nu_{L}$ on the kinetic equation for $f_{\alpha}$. (For $\nu_{L} \tau_{S D} / 3 \sim 1$, the slowing down distribution is strongly affected.) Given $\eta_{\alpha}$, we follow a recent work by D. Cohn. ${ }^{1}$ The bulk plasma power balance $3 n T / \tau_{E} \equiv P_{\text {loss }}=$ $P_{a u x}+\eta_{\alpha} P_{\alpha}$ and the definition $Q \equiv 5 P_{\alpha} / P_{\text {aux }}$ yields

$$
Q=\frac{5 P_{\alpha}}{P_{\text {loss }}}\left[1-\eta_{\alpha}\left(\frac{P_{\alpha}}{P_{\text {loss }}}\right)\right]^{-1}
$$

Defining

$$
\begin{aligned}
\left(n \tau_{E}\right)_{*} & \equiv 12 T /\left\langle\sigma_{f} v\right\rangle E_{\alpha o} \\
& \rightarrow P_{\alpha} / P_{\text {loss }}=n \tau_{E} /\left(n \tau_{E}\right)_{*}
\end{aligned}
$$

Note that $\left(n \tau_{E}\right)_{*}$ is a function of temperature, and if $\eta_{\alpha}=1$, achieving $n \tau_{E}=\left(n \tau_{E}\right)_{*}$ describes ignition. We rewrite Eq. (2) as

$$
Q=5 \frac{n \tau_{E}}{\left(n \tau_{E}\right)_{*}}\left[1-\eta_{\alpha} \frac{n \tau_{E}}{\left(n \tau_{E}\right)_{*}}\right]^{-1}
$$

and obtain Fig. 4.1, showing (a) how for a given normalized ignition margin $n \tau_{E} T /\left(n \tau_{E}\right)_{*} T, Q$ drops as $\eta_{\alpha}$ decreases below 1 , and (b) how, for a desired $Q,\left(n \tau_{E}\right) /\left(n \tau_{E}\right)^{*}$ has to be increased as $\eta_{\alpha}$ drops. From Eqs. (4.1) through (4.4), it thus becomes apparent how collective alpha effects producing an anomalous $D_{\alpha}$ could impact BPX performance and thus become detectable. The experimentally observed offset-linear dependence of plasma stored energy on heating power ${ }^{2}$ implies that the incremental stored energy at $Q=5 \mathrm{op}$ eration, relative to ohmic, should be twice that achieved with RF heating alone in a nonreacting plasma. $\eta_{\alpha}<1$ will be detectable as a reduced incremental confinement time, $\tau^{i n c}$, for alpha heating. Quantitative measurements of $\tau^{i n c}$ due to alpha particles require $Q \gtrsim 2$. 


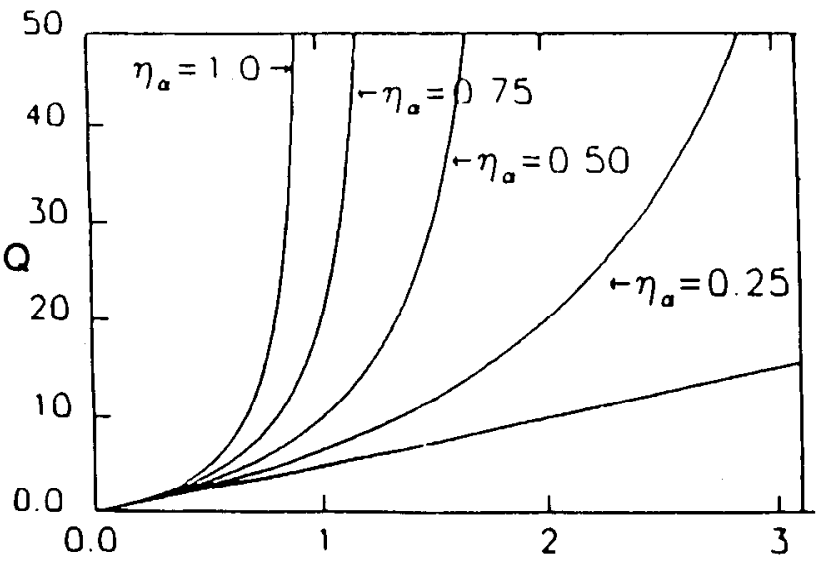

$\left(n \tau_{E}\right) /\left(n \tau_{E}\right) \cdot$

Fig. 4.1. $Q$ versus normalized ignition margin for different values of alpha coupling parameter $\eta_{\alpha}$. From Ref. 1.

\section{IV.C. EXPERIMENTAL RESULTS ON ALPHA SIMULATION AND ENERGETIC ION PHYSICS}

\section{IV.C.1. Single Energetic lon Confinement}

The fundamental benefit of fusion-born alpha particles in an axisymmetric tokamak is their ability to maintain the bulk plasma power balance via collisional slowing down against transport and radiation losses. It is therefore of keen interest to study confinement of energetic ions such as those produced in D-D and $\mathrm{D}-{ }^{3} \mathrm{He}$ plasmas in present large tokamaks. In fact, the fusion product gyroradii of these fuels are about the same as that for $\mathrm{D}-\mathrm{T}$ (but the relative concentrations are not: $n_{D D} / n_{e} \sim 10^{-4}$ versus $n_{\alpha} / n_{e} \leq 10^{-2}$ in D-T so that present D-D fusion products act merely as test particles). With the exception of certain so far unresolved triton burnup deficits, experimentation by Zweben et al. ${ }^{3}$ on TFTR shows agreement between actual confinement of D-D fusion products and classical guiding center orbit and collisional slowing-down theory in quiescent plasmas. ${ }^{4}$ Moreover, comparing the observed single-particle loss rate (coming mainly from near-transitionlayer counter-going ions) with calculations assuming anomalous diffusion coefficients of increasing magnitude, an upper bound of $D_{\alpha}<10^{3} \mathrm{~cm}^{2} / \mathrm{s}$ is found in TFTR, at $I_{p}=1.8 \mathrm{MA}$, compared to typical thermal ion diffusion of $D_{i} \simeq 2 \times 10^{4} \mathrm{~cm}^{2} / \mathrm{s}$. From this value of $D_{\alpha}$, the estimated fast alpha confinement time $\tau_{\alpha} \sim a^{2} / 4 D$ is much larger than the alpha thermalization time, thus allowing for self-heating of the fusion plasma.

These benign single-particle results are obtained in quiescent plasmas. When strong low-modenumber magnetohydrodynamic (MHD) fluctuations exist (in the range of $\tilde{B} / B_{0} \lesssim 10^{-3}$ driven by high neutral beam power). Zweben et al. ${ }^{5}$ observe $a \geq 3$-fold increase of fast ion losses to the detector, in phase with the MHD oscillations. Similarly, sawtooth crashes produce big bursts (three to four times bigger than classical first orbit losses) of lost ions, but with a small time-averaged effect. When the "super-Alfvénic" condition $v_{\| \text {fast }} \geq v_{\text {Alfven }}$ is added in a burning plasma, a new class of low- and high-mode-number MHD oscillations, the toroidal Alfvén eigenmodes (TAEs) may add to these losses (to be discussed below).

\section{IV.D. OBSERVATION OF COLLECTIVE OSCILLATIONS CAUSED BY ENERGETIC IONS}

Motivated by an increasing body of theoretical predictions of excitation of TAEs due to

$$
v_{\| \text {fast }}>v_{\text {Alfvén, }}
$$

parallel neutral beam injection (NBI) experiments satisfying this condition have recently been performed on DIII-D (Ref. 6) and TFTR (Ref. 7). For instability, besides the super-Alfvénic condition Eq. (4.5), the fast particle density gradient has to be steep enough (to yield $\omega_{* \text { fast }} / \omega \gtrless \frac{1}{2}$ ) and $\beta_{\text {fast }} / \beta_{\text {electron }}$ has to exceed a certain threshold. In both machines, when the magnetic field is reduced to $B \leq 1 \mathrm{~T}$ and other parameter optimizations are implemented to satisfy these three conditions, a new spectral feature is observed in the expected range of Alfvén frequency $\omega_{A}=k_{\|} v_{A}$, $k_{\|}=\left(2 R_{0} q\right)^{-1}$, at low mode numbers $n, m$. Moreover, the periodic bursts of this MHD activity are correlated with drops in the neutron signal of 5 to $7 \%$ in TFTR (see Fig. 4.2), and, in DIII-D, as much as $45 \%$ of the NBI power can be lost. However, in TFTR, $\epsilon \beta_{\text {pol }} \simeq \epsilon$, while in DIII-D, $\epsilon \beta_{\text {pol }}$ approaches the beta limit, which suggests a pressure-driven mechanism is involved besides the TAE mode (which by itself does not need a pressure gradient drive). Furthermore, in both machines, the critical beta for the onset of this instability is significantly larger than predicted by simple TAE theory (without including Alfvén continuum damping, not to mention nonlinear effects).

So far, no low-field, mainly parallel NBI experiments of this kind have been performed on JET. But ion cyclotron resonance frequency (ICRF) minority heating was used to produce $1-$ to $5-\mathrm{MeV}$ ${ }^{3}$ He ions, copious enough to contribute almost $50 \%$ of predominantly perpendicular energy, $P_{\perp} / P_{\|} \geq$ 10 to 50 . Thus, although $v_{\text {fast }} \simeq v_{\perp \text { fast }}=(2$ 


\section{IV.E. SPECIFIC THEORETICAL ALPHA LOSS MECHANISMS}

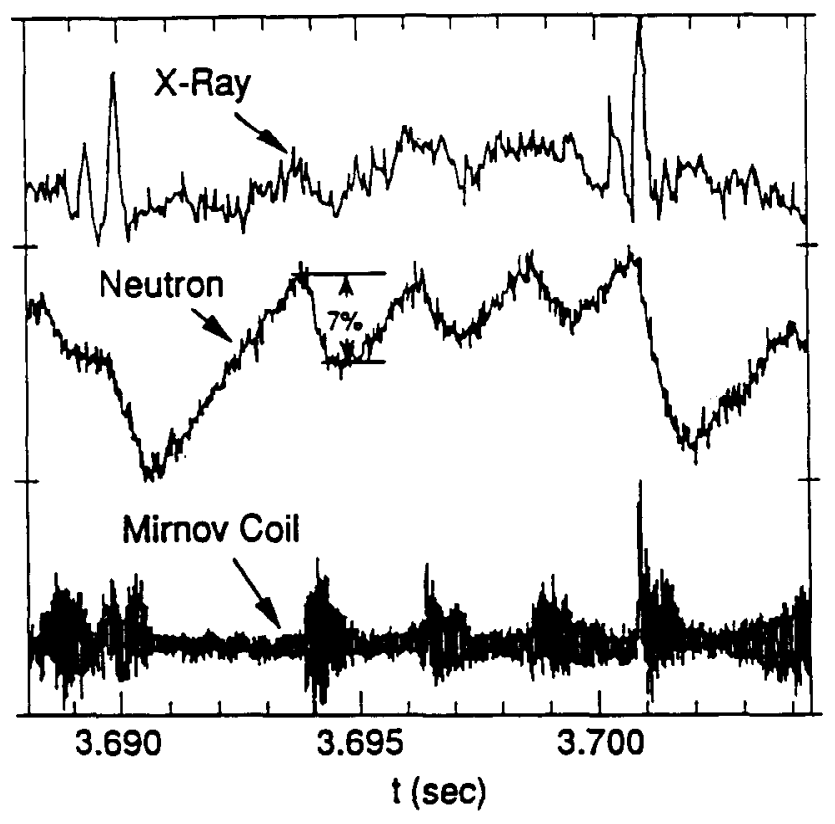

Fig. 4.2. TFTR neutron signal as a measure of ejection of $110-\mathrm{kcV}$ NBI ions, due to Alfvén wave excitation. From Ref. 7.

to 4) $v_{A}$, the shear Alfvén wave-particle resonance $\omega-k_{\|} v_{\| \text {fast }}-\boldsymbol{k}_{\perp} \cdot v_{D \text { fast }}=0$ is hardly populated. Under these conditions, slowing down is observed to be nearly classical with $\tau_{S D}<\tau_{\text {fast }}=$ $\left(4 D_{\text {fast }} / a^{2}\right)^{-1}$, where $D_{\text {fast }} \lesssim 0.2 \mathrm{~m}^{2} / \mathrm{s}$. In sawtooth crashes, $\sim 50 \%$ of fast ions are expelled from the $q=1$ region (but not necessarily from the plasma). Thus, overall, the results from JET (Ref. 8) are consistent with TFTR and DIII-D under overlapping conditions, but are lacking the contributions from the parallel-going fast ions.

Overall, the near-classical slowing-down behavior of TFTR, DIII-D, and JET observed in the absence of (a) large-amplitude Mirnov-like oscillations and (b) sufficient amounts of super-Alfvénic parallel-going ions is reassuring. However, underlying present bulk plasma fluctuation levels at $k_{\perp} \rho_{i} \leq 1$ are not entirely characteristic of possible Alfvén waves excited in future burning plasmas, which may include the shorter wavelengths $k_{\perp} \rho_{\alpha} \sim 1$ regime. Thus, the present results serve to provide the baseline in nonburning plasmas. D$T$ operation in TFTR and JET should reveal the location of some alpha stability boundaries, but BPX operating at $Q>5$ is needed to measure $\eta_{\alpha}$ quantitatively and thus determine the fusionrelevant fast alpha confinement.

\section{IV.E.1. Alpha Ripple Losses}

In TFTR, ripple losses have been analyzed ${ }^{9}$ numerically and experimentally. They are peaked at the outer midplane. At high current $\left(I_{p} \geq 1.5\right.$ MA), they are comparable in magnitude to prompt (first orbit) losses, but the stochastic threshold scales differently with $I_{p}, B$, and $\epsilon$ than prompt losses. The ripple loss time is much shorter than the slowing-down time.

In BPX so far, the 18-coil design with $R_{0}=2.6$ $\mathrm{m}, a=0.8 \mathrm{~m}$, and $q_{\psi}(a) \gtrless 3$ has been analyzed with respect to the dominant stochastic ripple losses using the RIPLOS code: At $R=R_{0}+a$, the toroidal field (TF) ripple is $<1 \%$ and $0.09 \%$ of the total alpha slowing down distribution is lost (Sec. V.L). The RIPLOS code simply and quickly evaluates the condition for the onset of stochastic ripple losses. An intermediate step, namely, following an ensemble of banana centers through their ripple loss and slowing-down history, is under development. The full treatment (requiring $>100$ hours of CRAY time or massively parallel computing) follows alpha-particle guiding centers. Such an effort is currently under way in a manner similar to that undertaken by the ITER physics team. However, comparisons of ripple amplitude between BPX and ITER (normalized to the stochastic and ripple-trapping thresholds) reveal that ripple losses should be comparable between the two devices. The full guiding center Monte Carlo ripple loss simulation is needed to provide detailed engineering information on the ripple loss power deposition profile on the first wall and physics information on the dependence of the ripple losses on the sawtooth inversion radius and the edge value $q_{\psi}(a)$ (Ref. 10). As long as $\tau$ (sawtooth) $\gg \tau_{S D}$, as expected, sawtooth-driven alpha ripple losses should be unimportant.

\section{IV.E2. Alpha Losses Due to Sawtoothing}

Experimentally, the results from TFTR, DIII$\mathrm{D}$ and JET were briefly discussed above, showing strong interaction with energetic ions. Theoretically, the interaction of alpha particles with the $m=n=1$ internal kink is still a very active topic, ${ }^{11,12}$ particularly where it concerns the explanation of the sawtooth period extension due to energetic particles (leading to "monster sawteeth"). In linear theory, retention of noncircular plasma cross section and of trapped particles of all pitch angles (including those near the trapped/circulating boundary) affects the stabil- 
ity results essentially and requires numerical treatment. ${ }^{13}$ Nonlinearly, it is now well recognized that in large, hot tokamaks such as JET, the sawtooth crash is much faster than predicted by Kadomtsev's resistive MHD model, but a new satisfactory model does not yet exist, even without the additional kinetic effects from the alpha particles. First, weakly burning plasma results from TFTR and JET D-T operation will provide guidelines, but obtaining the relevant BPX results at $Q>5$ and attempting sawtooth control may be pivotal for the operation of ETR-class tokamaks.

\section{IV.E3. Resonant Alpha Losses and Anomalous Alpha Diffusion Due to Alfvén Turbulence}

\section{IV.E.3.a. TAEs Destabilized by Super-Alfvénic Alpha Particles}

By the mid-1970s, Mikhailovskii et al. ${ }^{14}$ and Rosenbluth and Rosenbluth ${ }^{15}$ had discovered this fundamental low-mode-number instability mechanism (albeit for cylindrical Alfvén eigenmodes, worked out in detail by Tsang, Sigmar et al. ${ }^{16}$ ) with a growth rate of $10^{-2}$ of the Alfvén frequency. After the discovery of the toroidicity induced spectral gaps by Kieras and Tataronis ${ }^{17}$ and of destabilization of discrete modes in these gaps by C. Z. Cheng et al., ${ }^{18} \mathrm{Fu}$ and Van Dam ${ }^{19}$ demonstrated analytically an even faster growth rate of $2.5 \times 10^{-2} \times \omega_{A}$ in toroidal geometry, verified numerically by $C$. $Z$. Cheng's Nova-K code. ${ }^{20}$ The toroidal gap mode frequency is given by

$$
\omega_{r}=\left(\frac{v_{A}}{2 R_{0} q}\right)_{g a p}, q_{g a p}=\frac{m+\frac{1}{2}}{n},
$$

where $n$ and $m$ are the toroidal and poloidal mode numbers, and the gap location is determined from $k_{\|}^{2}(m)=k_{\|}^{2}(m+1)$. For a slowing-down distribution, the marginal stability criterion is of the form [see Eq. (37) of Ref. 20]

$$
\beta_{\alpha}\left[\frac{\omega_{* \alpha}}{\omega_{r}} F_{1}\left(\frac{v_{A}}{\bar{v}_{\alpha}}\right)-F_{2}\left(\frac{v_{A}}{\bar{v}_{\alpha}}\right)\right]=\beta_{e} \frac{v_{A}}{v_{t h e}},
$$

where $\omega_{* \alpha}=\left(\frac{m}{r}\right) \frac{\bar{E}_{\alpha} \partial n_{\alpha} / \partial r}{e_{\alpha} n_{\alpha} B}$ is driven by the energetic alpha density gradient and $\bar{E}_{\alpha} \equiv m \bar{v}_{\alpha}^{2} / 2$ is the fast alpha energy averaged over the alpha distribution function. The functions $F_{1,2}\left(v_{A} / \bar{v}_{\alpha}\right)$ originate from inverse and regular Landau damping on the alpha particles and electrons. ${ }^{20}$ Equation (4.7) is obtained from an analytic large-aspectratio dispersion relation localized in radius around the gap mode peak. The more exact marginal stability curve obtained (numerically with the Nova-K

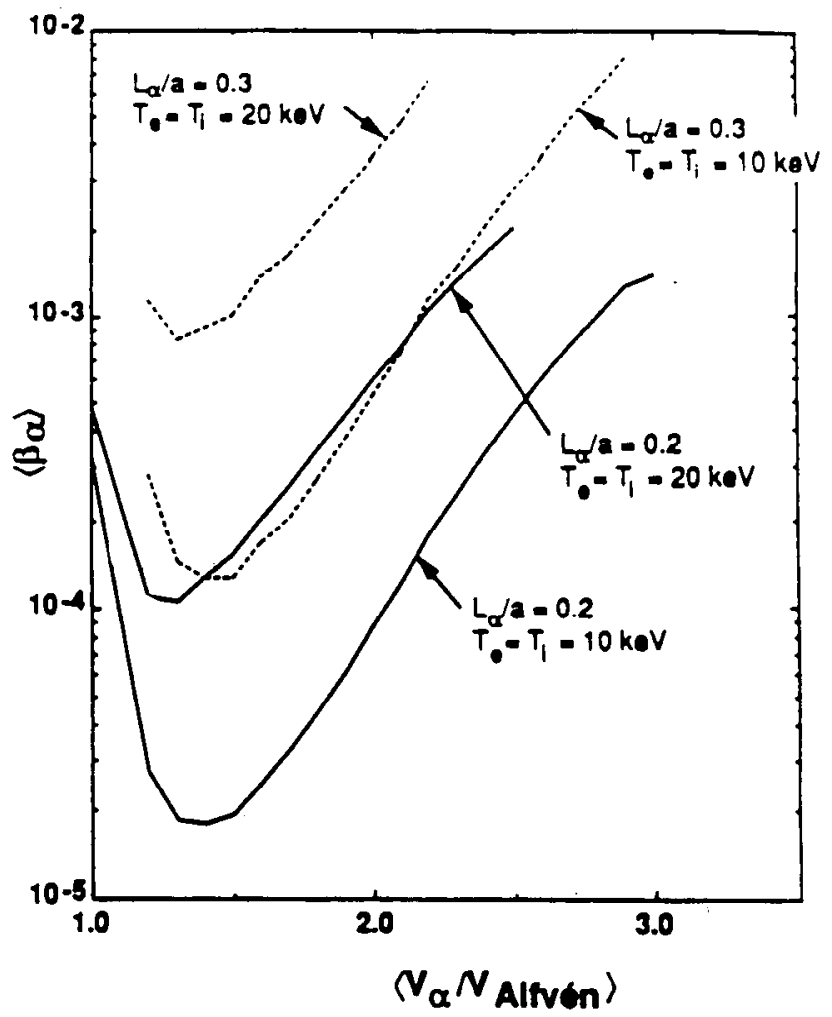

Fig. 4.3. Global eigenvalue calculation delineating the TAE stability boundary at fixed $T$ for standard BPX equilibrium. $L_{\alpha} / a$ is the ratio of alpha density gradient scale length to minor radius. ${ }^{20}$

code) from a global stability calculation is shown in Fig. 4.3 for BPX equilibrium parameters. In this calculation, $n_{\alpha}=n_{\alpha}(0) e^{-r^{2} / L_{\alpha}^{2}}$ is assumed, and results are shown for $L_{\alpha} / a=0.2$ and 0.3 for fixed temperatures $T_{e o}=T_{i o}=10$ and $20 \mathrm{keV}$. ( $a$ is the minor plasma radius.) In this figure, the volume-averaged $\left\langle\beta_{\alpha}\right\rangle$ and the ratio of alpha birth speed to Alfvén speed, $v_{\alpha} / v_{A}$, are free parameters entering the kinetic part of the Nova-K code, for a fixed MHD equilibrium, producing the Alfvén gap mode spectrum. The toroidal mode number is fixed at $n=1$; the poloidal spectrum centers about $m=1$ and 2 . The plasma is stable below each curve and unstable above. The sharply rising boundary on the left comes from the left-hand side of (4.7), i.e., the condition $\omega_{* \alpha} / \omega_{r}>F_{2} / F_{1}$ for instability, where $F_{2} / F_{1} \sim O(1)$. By increasing $v_{A} \sim B / \sqrt{n_{i}}$, one can thus avoid instability, but at $B=9 \mathrm{~T}, v_{\alpha o}<v_{A}$ requires $n_{i}<0.57 \times 10^{20} \mathrm{~m}^{-3}$ (for a 50:50 D-T plasma), which is below the expected operating region. Concerning $\beta_{\alpha}$, at fixed $\beta_{\text {thermal }}=3 \%, \beta_{\alpha}=1.5 \times 10^{-3}$ at $10 \mathrm{keV}$ and $\beta_{\alpha}=0.01$ at $20 \mathrm{keV}$ (cf. Fig. 3 of Ref. 21). Thus, 


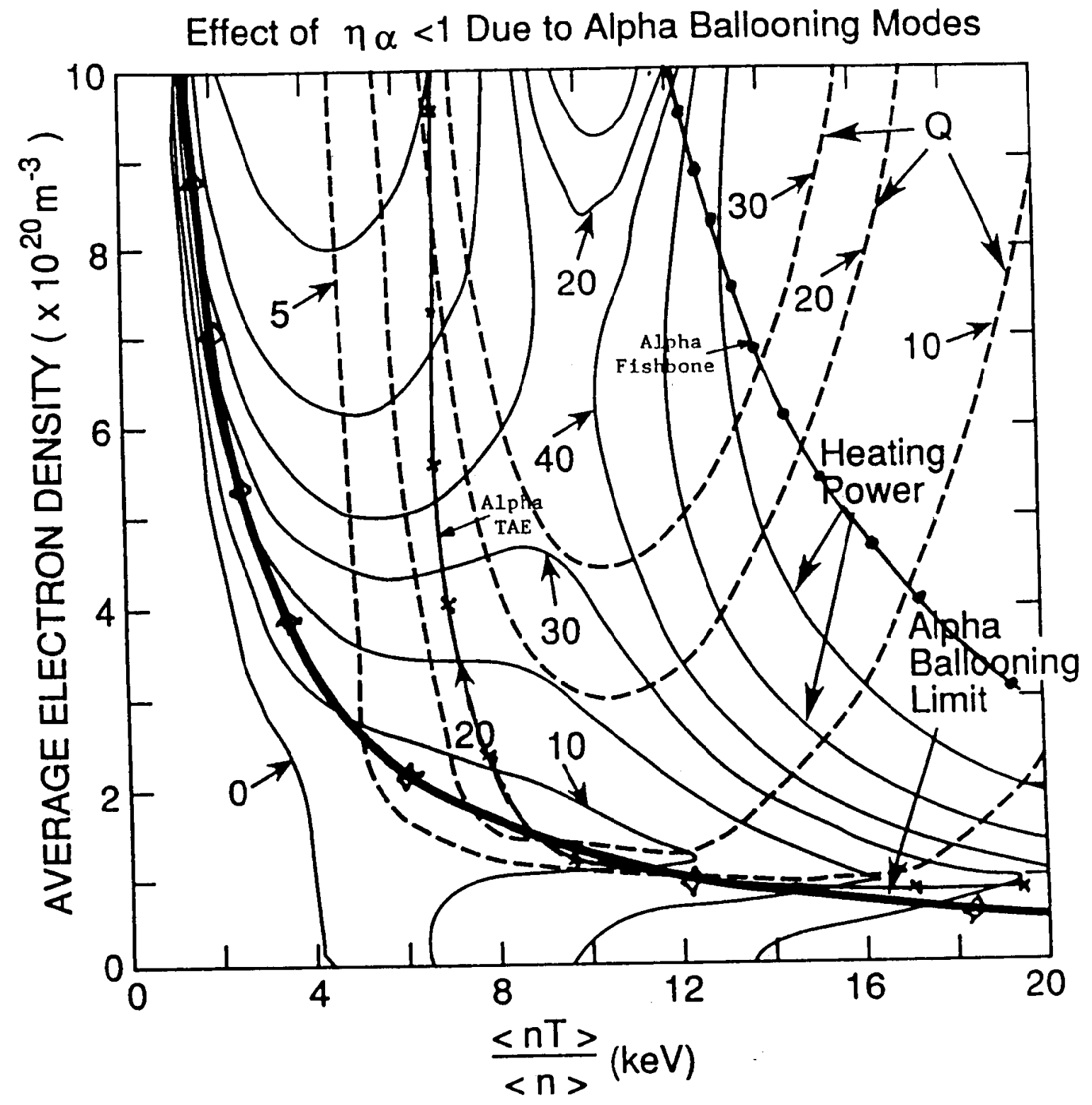

Fig. 4.4. Various alpha-driven instability boundaries superimposed on POPCON plot for BPX. Heavy line with diamonds: onset of alpha ballooning driven alpha diffusion; line with xxx: alpha-driven TAE; line with circles: alpha fishbones for $\left(r q^{\prime} / q\right)_{1}=0.2$ 
the linear stability condition Eq. (4.7) can be violated in BPX, depending on the operating point (see Fig. 4.4). Still under investigation is the linear damping of the alpha-driven TAE due to a stabilizing coupling effect of the main gap mode to continuum modes near the plasma edge.22

An important additional reduction in growth rate has recently been discovered ${ }^{23}$ to come from the poloidal harmonic side bands at $v_{\|}=v_{A} / 3$. This enables thermal ion Landau damping to exceed electron Landau damping by a factor of $\sim 5$, overall (for $\beta_{i}=2 \%$ ).

Linearly, broadening of the alpha density profile is stabilizing. For typical $n$ and $T$ profiles, $L_{\alpha}$ (birth) $/ a \geq 0.20$. If one assumed that the nonlinear consequence of the TAE-induced anomalous alpha transport was to broaden $n_{\alpha}(r)$ until it decreased $\omega_{* \alpha}$ enough to satisfy Eq. (4.7), a simple estimate is $L_{\alpha}$ (marginal) $/ a \simeq 0.5$. Such an $n_{\alpha}(r)$ profile implies modest changes in the alpha power deposition, but there is no clear reason to expect such a benign saturation mechanism rather than, for example, strong relaxation oscillations similar to those observed in beam-driven fishbone modes, which can substantially deplete the beam ion population.

Besides quasi-linear profile broadening and flattening of the resonance region in velocity space, ${ }^{24}$ alpha orbit losses due to the finite-amplitude TAE waves $^{25}$ have been under investigation. With regard to the latter, a subsequent rigorous analysis of single alpha-particle guiding center losses in action angle variables as well as Monte Carlo simulations of an ensemble of fast alpha particles have been done at prescribed $\tilde{B}_{r} / B_{o} \geq 10^{-4}$. In recent work by Sigmar and $\mathrm{Hsu},{ }^{26}$ the simulation is arranged such that prompt loss orbits are entirely removed from re-entering the statistics. Qualitatively, the earlier results of Ref. 25 are confirmed. The resonance condition $\omega-k_{\|} v_{\| \alpha}-k_{\perp} v_{D \alpha}=0$, where $\omega=\omega_{\text {gap }}$ is shown to be maintained for several alpha transit times during which the particle loses energy to the wave, for realistic tokamak geometry and radial TAE mode structure. During resonance periods, the alpha particles suffer a secular radial drift that scales $\propto \tilde{B}_{r} / B_{o}$. Over longer periods they experience a random walk in constants of motion space $\left(E, \mu B_{o} / E, P_{\phi}\right)$ which scales $\propto\left(\tilde{B}_{r} / B_{o}\right)^{2}$. Figure 4.5 shows an alpha orbit Poincaré plot in $P_{\varphi}-\varphi$ space (where $\varphi$ is the toroidal angle). Thirty-two monoenergetic passing alpha particles are launched with $E_{0}=3.52$ $\mathrm{MeV}$, magnetic moment $\mu_{0}=0$, and equal spacing of $P_{\varphi 0}$ initially. (Higher/lower $P_{\varphi}$ corresponds to inner/outer radial position.) The $n=1, m=$ $0,1,2,3$, components of the imposed TAE distur- bance with amplitude $\tilde{B}_{r} / B_{0} \simeq 2 \times 10^{-3}$ produce orbit stochasticity above the KAM surfaces and island regions below. We note that although strong stochasticity appears in the upper $P_{\varphi}$ (i.e., small plasma radius) region, there is no stochastic particle loss across the plasma boundary that corresponds to a location below the KAM surfaces. However, with $\mu B_{0} / E_{0} \sim O(1)$, the same condition will lead to a fully stochastic $P_{\varphi}-\varphi$ Poincaré plot and radial losses. (The TAE obeying the $E_{\|}=0$ constraint of ideal MHD does not stochasticize the magnetic field lines, only the alpha orbits.)

Stochastic orbit overlap occurs at $\tilde{B}_{r} / B_{0}=2 \times$ $10^{-3}$ for $n=1$ and at lower amplitudes when more than one toroidal mode number is admitted. For one $n$, the phase-space diffision coefficient $D_{p_{\phi} p_{\phi}} \propto I_{p}^{-2}$, i.e., a favorable scaling with plasma current. Several gap modes indeed coexist experimentally, ${ }^{7}$ semilocalized at different radii, depending on the profiles of $\omega_{A}^{2} \equiv k_{\|}^{2} v_{A}^{2}$, where $R_{o} k_{\|}=n-m / q(r)$. For example, when the ion density (contained in $v_{A}^{2}$ ) varies as $n_{i} \sim[q(r)]^{-2}$, a channel of frequency gaps can open from the plasma center to the edge without encountcring a continuum branch near the edge (which could act as a damping mechanism ${ }^{21}$ ). Quantitative results of Monte Carlo modeling of alpha phase-space diffusion into the prompt orbit loss region are underway. Much more work (including improvements in linear continuum mode damping, nonlinear saturation, and calculations of a self-consistent $D_{\alpha}$ ) is needed before the total impact of TAE on BPX can be predicted theoretically.

\section{IV.E.3.b. Alpha Ballooning Modes}

The standard ballooning stability threshold can be theoretically lowered by fast alpha particles. For trapped alpha particles, the precession resonance is destabilizing, for circulating alpha particles, the wave frequency-transit frequency resonance acts similarly. Earlier work ${ }^{27}$ has recently been extended to a high-frequency branch $\omega_{r} \simeq \omega_{\text {Alfven }}$ for high mode numbers. The low-frequency branch. $\omega_{r} \sim \omega_{* i}$ can be stabilized when the fast alpha banana precession frequency exceeds $\omega_{r}$. The highfrequency alpha-driven branch is unstable. It has a simplified (local) $\beta_{\alpha}$ threshold valid for $\epsilon v_{A} / v_{\alpha} \geq$ $k_{\theta} \rho_{\alpha} \geq L_{\alpha} / R_{o} q$ :

$$
\beta_{\alpha}^{c r i t} \simeq f_{r}^{-1} \frac{v_{\alpha}}{v_{A}}\left(\beta_{e} \frac{m_{e}}{m_{i}}\right)^{1 / 2}
$$

where $f_{r}$ is the fraction of resonant alpha particles. For $v_{\alpha} / v_{A}=1.5$, this gives typically $\beta_{\alpha}^{\text {crit }} \simeq 10^{-3}$, a 


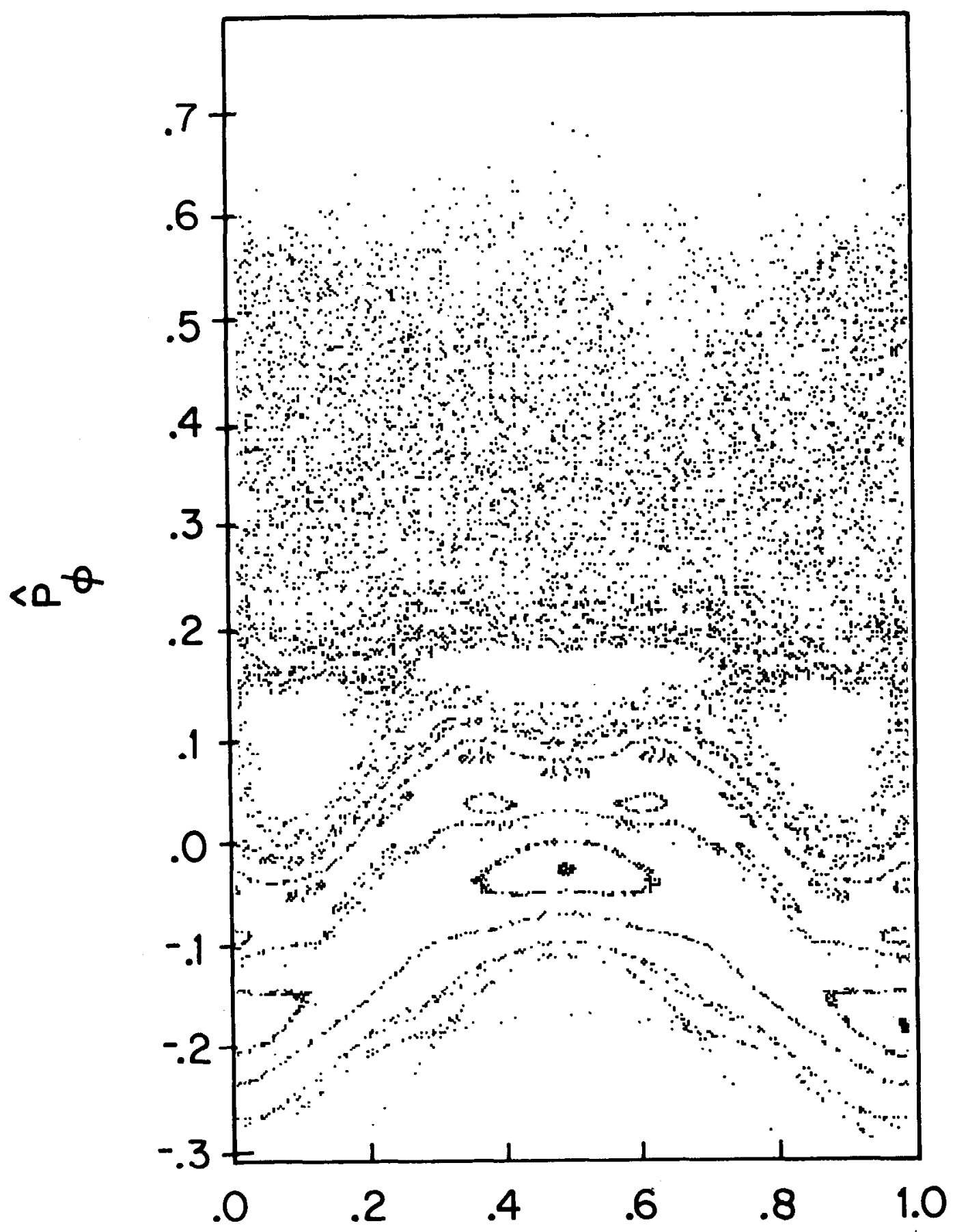

$\hat{\phi}$

Fig. 4.5. Alpha orbit Poincaré plot $P_{\varphi}$ vs. $\varphi$, driven by TAE of amplitude $\tilde{B}_{r} / B_{0}=2 \times 10^{-3}$, with mode numbers $n=1, m=0,1,2,3$. From Ref. 26 . 
value easily achieved in BPX. However, this high$n$ mode is not expected to produce a hard beta limit but a soft onset of fast-alpha diffusion. Using quasi-linear theory, Rewoldt estimates in Ref. 27 that $D_{\alpha} \sim \chi_{e}$ on the ballooning unstable flux surfaces. In Ref. 27 it was shown that in terms of the critical background beta, $\beta_{t h}^{\text {crit }}$, the alpha ballooning threshold is exceeded for $\left(\beta_{\text {th }}^{\text {crit }} / 4\right)$. This is indicated in Fig. 4.4 as the curve labelled "alpha ballooning." Experimentally, the Alfvén frequency excitation observed in DIII-D (Ref. 6) appears to have a pressure-driven component with toroidal mode numbers 4 to 9 , which could be a form of fast-ion-driven ballooning modes.

A specific analysis for BPX with radial profiles profiles of $n, T$, and $q$ taken from a run of the BALDUR radial transport code simulating $\mathrm{H}$-mode operation in BPX is under way. The radial density profiles for the background species are very flat in the interior, that for alpha particles is very steep. In addition to the $\omega_{r} \sim \omega_{* p i}$ root, another unstable branch is found with a larger linear growth rate, which is a high- $n$ extension of the low- $n$ TAE. The new $\omega_{A}$ root is destabilized by increasing steepness of the alpha-particle profile.

\section{IV.E.3.c. Alpha Fishbone Oscillations}

The linear theory and threshold calculations have been readdressed recently. ${ }^{11,12}$ Following Ref. 11, the threshold value for the onset of alphadriven fishbones is $\beta_{\alpha, \text { trapped }}^{\text {crit }}=\epsilon\left\langle\omega_{D \alpha}\right\rangle / \omega_{A}$, where $\left\langle\omega_{D \alpha}\right\rangle=v_{\alpha}^{2} q / 2 r R \Omega_{\alpha}, \omega_{A}=v_{A} / \sqrt{3} R\left(r q^{\prime} / q\right)_{1}$. (We note that this is really a density, not a beta limit.) Here $\Omega_{\alpha}$ is the alpha gyrofrequency, and the shear parameter $s=\left(r q^{\prime} / q\right)_{1}$ is evaluated at the $q=1$ resonant surface. $s$ cannot be measured easily and is assumed here to be $s \approx 0.2$ [depending on $q(o)$ and $\left.r_{1} / a \simeq q(a)^{-1}\right]$. This formula for $\beta_{\alpha}^{c r i t}$ was derived analytically in the limit of deeply trapped particles. Inserting the present BPX parameters yields $\beta_{\alpha}^{\text {crit }} \simeq 10^{-2} v_{\alpha o} / v_{A}$. [For a fuller display of this threshold cf. Eq. (40) in Ref. 11]. For $B=9 \mathrm{~T}$, $n_{20}=3, v_{\alpha o} / v_{A}=2$ and $s=0.2, \beta_{\alpha}^{c r i t} \simeq 2 \%$, which is not reachable with thermalized fusion even at $T>20 \mathrm{keV}$ (see Fig. 4 or Fig. 3 of Ref. 21). However, there are indications in JET that $s$ at the $q=1$ surface may be considerably flatter than 0.2 . In that case, BPX may be able to enter the alphadriven fishbone regime, particularly if the contributions from alpha-pitch angles other than deeply trapped ones play an additional destabilizing role. This may be explorable in present JET plasmas with ICRH-produced energetic trapped particles if the ICRH power is sufficient to cross the $\beta_{\text {hot }}$ threshold.

\section{IV.E.3.d. Summary of Collective Alpha Phenomena}

To summarize, Fig. 4.4 shows all these marginal onset curves in a BPX POPCON diagram. Notice the soft onset of alpha ballooning at $\frac{1}{4} \beta_{\text {Troyon, }}$, the theoretical TAE threshold, and the alpha fishbone threshold (which could come down in $\beta_{\alpha}$ if the shear at the $q=1$ surface is flatter): Overall, BPX appears capable to pass through these thresholds (if they exist self-consistently) and to explore their importance for the burning plasma performance. It is important to note that the divertor system in BPX is not anticipated to be capable of handling the power outflux at the beta limit, at 9 T. However, at modestly lower fields, alpha particle studies near the beta limil are possible (since $\left.P_{f u s} \propto \beta^{2} B^{4}\right)$ if confinement is slightly favorable, or the heating system is upgraded. Because alpha instabilities are anticipated in some regions of parameter space, BPX wall armor and protective limiters must be designed to handle a substantial load of alpha power, to permit steady operation in these unstable regimes. As an example, one could imagine attaining $Q \sim 10$ at $\eta_{\alpha} \sim 0.5$, which would imply an alpha loss power of $\sim 20 \mathrm{MW}$ with 20 MW of auxiliary heating. Further calculations will be required to optimize limiter and first-wall design to handle this power flux.

\section{IV.F. THERMAL STABIUTY}

Depending on available $P_{\text {aux }}$ and on the achievable H-mode improvement factor on $\tau_{E}$ (where it has been shown elsewhere in this paper that 1.85 $\times$ ITER-P is expected and even a small increase to 1.95 would produce a sizable increase in Q) BPX may cross the thermal stability threshold which basically follows the ridge in $n-T$ space leading to the Cordey pass, see Fig. 4.6. ${ }^{a}$ For this case, $Z_{\text {eff }}=1.65, \tau_{E}=1.85 \times$ ITER-P and for the profiles $n \sim T \sim(1-\psi)$ was assumed. Note that in this diagram, the central density and temperature values are plotted (versus $\langle n\rangle$ and $\langle n T\rangle /\langle n\rangle$ in the standard POPCONS). It will be worthwhile in BPX to explore indications of thermal selfstabilization due to the various anomalous fast alpha diffusion mechanisms discussed above, which reduce the self-heating efficiency $\eta_{\alpha}$, thereby regulating the operating point.

\footnotetext{
'This figure was provided by E. A. Chaniotakis, Plasma Fusion Center, Massachusetts Institute of Technology (1990)
} 


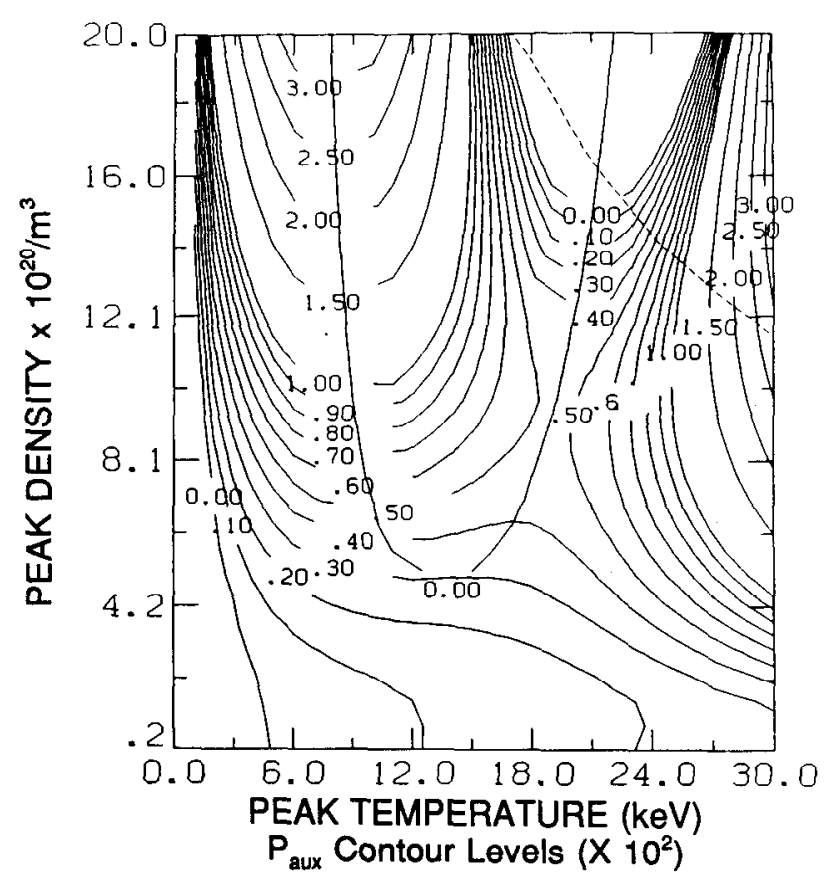

Fig. 4.6. Thermal stability marginal boundary (line with crosses) for a BPX equilibrium with profiles $n \sim T \propto 1-r^{2} / a^{2}, Z_{\text {eff }}=1.65, \tau_{E}=1.85 \times$ ITER-P. Also shown are the $P_{\text {aux }}$ contours $\left(\times 10^{2}\right.$, in MW). Courtesy of E. A. Chaniotakis, 1990.

\section{IV.G. HELIUM ASH}

At full-fusion-power wall-load values corresponding to $P_{\text {loss }}=100 \mathrm{MW}$, the flattop time for the loss power is $3 \mathrm{~s}$, preceded by a 5 -s rampup. In this relatively short time, the helium ash accumulation $n_{\mathrm{He}} / n_{e}$ is between 2 and $4 \%$, enough to be discernible, but not enough to quench the burn. This is shown in a zero-dimensional timedependent simulation by Stotler (Sec. III.D) assuming $\tau_{E}=2 \times \tau_{E}$ (ITER-P), $P_{a u x}=20 \mathrm{MW}$, and $Z_{\text {eff }}=1.65$ (see Fig. 4.7). This simulation is identical to that of Fig. 3.25 of Sec. III.D.3 except that the helium ash from fusion reactions is allowed to accumulate (no losses) with the initial helium number adjusted so that $n_{\mathrm{He}} / n_{e}=1.7 \%$ at the start of heating, easily consistent with ion cyclotron minority concentration requirements. The helium density profile is assumed to be identical to that of the hydrogenic species (square-root parabolic). Note the drop of $P_{\alpha}$ after $t=11 \mathrm{~s}$ due to helium accumulation. At the peak of $P_{\alpha}, n_{H e} / n_{e}=3.5 \%$. Thus, BPX can reveal whether this effect exists. Anomalous outward transport of epithermal alpha particles could be a natural consequence of the fusionenhanced fluctuation spectrum, an effect that may not exist in $Q \lesssim 1$ machines. If helium ash should indeed accumulate centrally, BPX may be able to

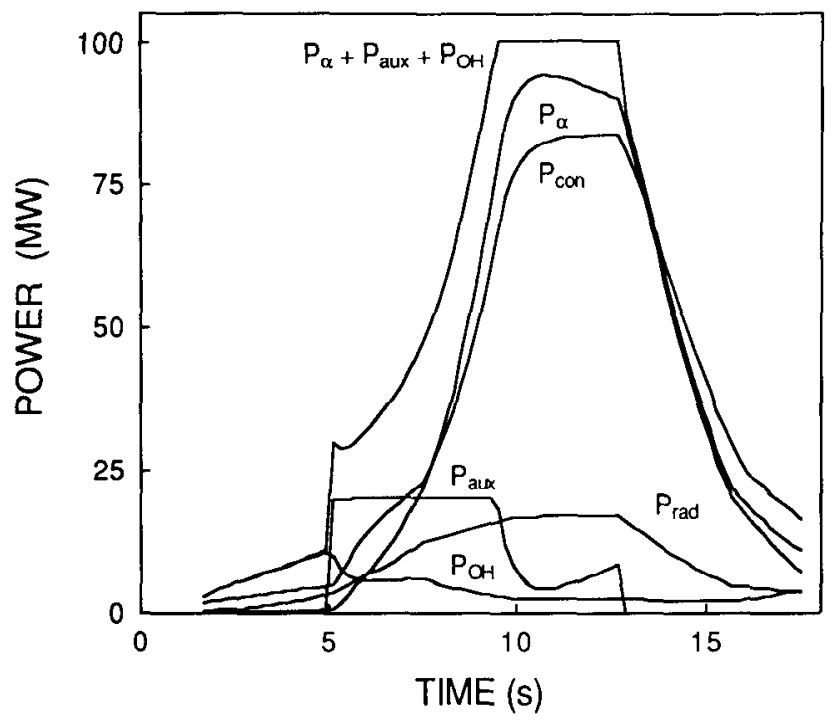

Fig. 4.7. Zero-dimensional time-dependent simulation of $P_{\alpha}$ evolution including helium ash accumulation. For $t>11 \mathrm{~s}$, fuel dilution begins to affect the fusion burn. (From D. Stotler, Sec. III.D.)

use its ICRF equipment to apply Chang's theory of resonant ash removal. ${ }^{28}$

\section{IV.H. SUMMARY AND CONCLUSIONS}

As indicated in Fig. 4.1 and various POPCON plots, BPX can explore, over a wide range in $n-T$ space, the effects of less-than-classical alpha power coupling efficiency $\eta_{\alpha}$. It can determine the impact of self-consistent alpha ripple transport and alpha-driven fluctuations on bulk plasma stability as well as on bulk plasma energy and particle confinement. This experiment will be the first to enter the new regime of $P_{\alpha}>P_{a u x}$, simultaneously containing an isotropic fast alpha distribution $f_{\alpha}$, with a fully populated $v_{\| \alpha}>v_{\text {Alfvén }}$ component. Across its available density and temperature range, BPX can move in and out of alpha-instability areas, e.g., alpha-TAE, alpha-ballooning, alpha-fishbones, and alpha-driven sawtooth oscillations. Furthermore, it may be able to cross thermal stability boundaries and reveal potential self-stabilization due to alpha-induced losses affecting $\eta_{\alpha}$ and possibly bulk plasma confinement. BPX can explore incipient helium ash accumulation in the presence of a fully developed alpha-driven fluctuation spectrum (which may provide anomalous outward diffusion). Finally, BPX will show the level of sustainable con- 
finement enhancement over $\mathrm{L}$ mode and the required H-mode power threshold in the presence of strong central isotropic alpha heating. Central power deposition may produce better than the currently known confinement scaling, ${ }^{29}$ but at the present state of understanding of plasma energy confinement, it remains for the experiment to reveal the additional impact of the fully developed alpha particle component on plasma energy confinement.

\section{REFERENCES}

1. D. COHN, PFC/JA-9-6, Massachusetts Institute of Technology, submitted to J. Fusion Energy.

2. M. BELL et al., Plasma Phys. Controlled Fusion, 28, 1329 (1986).

3. S. ZWEBEN et al., Nucl. Fusion, 30, 1551 (1990).

4. S. ZWEBEN et al., Princeton Plasma Physics Laboratory report PPPL-2770 (1991) accepted for publication in Nuclear Fusion.

5. S. ZWEBEN et al., Proc. 16th European Conference Fusion Plasma Physics, Venice, Italy, March 13-17, 1989.

6. W. HEIDBRINK et al., Nucl. Fusion, 31, 1635 (1991).

7. K. L. WONG et al., Phys. Rev. Lett., 66, 1874 (1991).

8. G. COTTRELL et al., Nucl. Fusion, 31, 61 (1991).

9. R. BOIVIN et al., Ph.D. Thesis, Princeton Plasma Physics Laboratory report PPPL-2797 (1991).

10. K. TANI et al., Proc. 12th Int. Conf. Plasma Physics and Controlled Nuclear Fusion Research, Nice, France, June 20-24, 1988, Vol. 2, p. 121, International Atomic Energy Agency (1988).

11. R. B. WHITE, F. ROMANELLI, and M. BUSSAC, Phys. Fluids, 32, 745 (1990).

12. B. COPPI et al., Phys. Fluids B, 2, 927 (1990).
13. C. Z. CHENG et al., Proc. 13th Int. Conf. Plasma Physics and Controlled Nuclear Fusion Research, Washington, D.C., October 1-6, 1990, Vol. 2, p. 209, International Atomic Energy Agency (1990).

14. A. MIKIIAILOVSKII, Zh. Eksp. Teor. Fiz., 68, 1772 (1975); Sov. Phys. JETP, 41, 890 (1975).

15. M. ROSENBLUTH, and P. RUTHERFORD, Phys. Rev. Lett., 34, 1428 (1975).

16. K. TSANG, D. J. SIGMAR, and J. WHITSON, Phys. Fluids, 24, 1508 (1981).

17. C. KIERAS and J. TATARONIS, J. Plasma Phys., 28, 395 (1982).

18. C. Z. CHENG, L. CHEN, and M. S. CHANCE, Ann. Phys., 161, 21 (1984).

19. G. Y. FU, and J. VAN DAM, Phys. Fluids $B$, 1, 1949 (1989).

20. C. Z. CHENG, Phys. Fluids, B 3, 2463 (1991).

21. H. P. FURTH, R. J. GOLDSTON, S. J. ZWEBEN, and D. SIGMAR, Nucl. Fusion, 30, 777 (1990).

22. F. Zonca et al., Princeton Plasma Physics Laboratory report PPPL-2799 (1991) submitted to Phys. Rev. Lett.

23. R. Betti and J. Freidberg, Massachusetts Institute of Technology report PFC/JA-91-25 (1991), submitted to Phys. Fluids.

24. H. BERK and B. BREIZMAN, Phys. Fluids $B$, 2, 2246 (1990).

25. D. SIGMAR, C. T. HSU, C. Z. CHENG, and R. E. WHITE, PFC/JA/89-8, Massachusetts Institute of Technology (1989), submitted to Phys. Fluids, (1991).

26. D. SIGMAR and C. T. HSU, submitted to Phys. Fluids, (1991).

27. G. REWOLDT, Phys. Fluids, 31, 3727 (1988). 28. C. S. CHANG et al., Phys. Fluids $B, 2,2383$ (1990).

29. R. WALTZ, Proc. 13th Int. Conf. Plasma Physics and Controlled Nuclear Fusion Research, Washington, D.C., October 1-6, 1990, Vol. 2, p. 273, International Atomic Energy Agency (1990). 